\title{
Research into a real-time in-situ sensor-based monitoring concept for biaxial stress monitoring in textile architecture
}

\author{
Sarah Stipek*, Mathis Bruns ${ }^{\dagger}$, Mike Ludwig ${ }^{\dagger 1}$, Andreas Nocke ${ }^{\dagger}$ and Chokri Cherif ${ }^{\dagger}$ \\ * TU Dresden \\ Institute of Textile Machinery and High Performance Material Technology \\ Dresden, Germany \\ e-mail: sarah.stipek@mailbox.tu-dresden.de \\ ${ }^{\dagger}$ TU Dresden \\ Institute of Textile Machinery and High Performance Material Technology \\ Dresden, Germany \\ ${ }^{\dagger 1}$ dresden elektronik ingenieurtechnik gmbh \\ Dresden, Germany
}

\begin{abstract}
In textile architecture, prestressed surface structures are influenced, for example, by incorrect construction, structural defects of the material or strongly varying weather influences and ageing effects [1]. These uncertainties lead to very high safety factors in the design and shortcycle manual monitoring measures, which predominantly result in a subjective assessment of the built membrane structure. The monitoring methods used so far are for example based on a stationary deformation measurement, stress-based reverse calculation methods of the primary structure (monitoring of cable stresses) or on a manual and local strain or material stress measurement with handheld devices. In order to guarantee the designed prestress during the erection phase on the one hand and to be able to react to the reduction of the designed prestress in the large-area membrane surface in real-time over the lifetime on the other hand, a network of resistive yarn-based strain sensors will be applied, which continuously detect the membrane strains and make them available for the required mechanical stress calculation. A previous research has proven, that yarn-based strain sensors can detect the biaxial non-linear, orthotropic and visco-elastic material strain behaviour of a main base material (PVC-coated polyester fabric) in textile architecture.

Therefore, this paper focuses primarily on the integration of yarn-based strain sensors into the manufacturing process of a membrane structure and the real-time monitoring of the stress distribution over the lifetime.
\end{abstract}

\section{ACKNOWLEDGMENT}

The authors would like to express appreciation for the support of the Zentrales Innovationsprogramm Mittelstand (ZIM) of the Bundesministerium für Wirtschaft und Energie (BMWi), [Project ZF4006510TS8].

\section{REFERENCES}

[1] Uhlemann, J., Elastic constants of architectural fabrics for design purposes. Doctoral Dissertation, Universität Duisburg-Essen, Fakultät für Ingenieurwissenschaften» Bauwissenschaften» Bauingenieurwesen., (2016). 